\title{
Temperature measurement by infrared thermography in a lubricated contact : radiometric analysis.
}

by REUNGOAT D. and TOURNERIE B.

Laboratoire de Mécanique des Solides, URA CNRS 861, Université de Poitiers.

\section{Abstract}

A method of surface temperature measurement by infrared thermography in a lubricated contact has been developed. The capabilities of the camera are examined. The analysis, which needs the determination of global radiometric coefficients and leads to radiometric equations is presented. The calibration of emissivity and transmittivity coefficients of the involved materials is reported. The validity of the method is tested and discussed.

\section{Nomenclature}

E global coefficient of emission

$L$ radiant intensity

$M$ radiative power

$\mathbf{R}$ global coefficient of reflection
T global coefficient of transmission

$T$ temperature

$\varepsilon$ coefficient of emissivity

$\rho$ coefficient of reflectivity

$\tau$ coefficient of transmittivity subscripts

0 blackbody

$\mathrm{i}, \mathrm{j}, 1,2,3,4$ different media

+ , - direction of propagation

\section{Introduction}

The temperature in the interface of a lubricated contact is an important parameter since it is dependent on friction losses and it controls the lubricant viscosity and the thermoelastic distortions of the surfaces. The experimental determination of the field of temperature in the interface gives data which are very useful to validate numerical models. Infrared thermography is the best technique to determine face temperature without contact on rotating machine elements and to give temperature mapping of the scanned area, but few attempts have been made to introduce this measurement technique in lubrication and tribology [1] , [2] , [3] .

\section{Features of the infrared camera}

The infrared camera used in this study is the AGEMA $880 \mathrm{SW}$ model. A $12^{\circ}$ lens has been adapted to the camera through a setting ring in order to magnify the image of the scanned surface.

The nonlinear relation between the blackbody object temperature $T$ (which emits a radiative power $M_{0}$ measured by the detector) and the instrument read-out $U$, is given by the calibration function of the camera $f\left[U, M_{0}(T)\right]=0$. This function is used to determine the blackbody object temperature $T$ for a known value of the read-out instrument $U$ but also to calculate the output value $U$ due to the radiative power $M_{0}$ emitted by a blackbody at a known temperature $\mathrm{T}$.

The slit response function has been measured in laboratory conditions; for $98 \%$ of modulation the elementary angle of measurement is of 0.0058 radian, that correspond to 29 points of measurement by scanned line. In the conditions of the experiments the noise equivalent temperature difference has been established; it is less than $0.12^{\circ} \mathrm{C}$ for temperatures greater than $20^{\circ} \mathrm{C}$. 
http://dx.doi.org/10.21611/qirt.1994.006

\section{Radiometric analysis}

\subsection{Description of the model}

The studied lubricated contact is shown in figure 1. It consists of;

- a rotating annular surface of a ring made of graphite,

- a static plate of Calcium Fluoride applied against the rotor,

- an oil film, whose thickness is of some micrometers, which separates the two surfaces of contact.

The sliding speed is parallel to plane of contact.

The infrared radiation emitted by the carbon surface is transmitted to the camera in a direction perpendicular to the interface through three semi-transparent media; the oil film, the Calcium Fluoride plate and the atmosphere.

\subsection{Global coefficients of the semi-transparent media}

The analysis is based on the following assumptions:

- there is no diffusivity,

- the media are isotropic,

- the absorption coefficient and the temperature are constant along the thickness.

The emissivity and the transmittivity of the semi-transparent media are dependent on the spectral band of the camera. The mathematical integration of these coefficients is complex, and in this study they have been experimentally calibrated as it is explained in the following section.

The reflectivity $\rho_{i, j}$ and the transmittivity $\tau_{i, j}$ of the dioptre formed by the interface between the two semi-transparent media $i$ and $j$ are given by;

$$
\rho_{i, j}=\frac{\left(n_{j}-n_{i}\right)^{2}+\left(k_{j}-k_{i}\right)^{2}}{\left(n_{j}+n_{i}\right)^{2}+\left(k_{j}+k_{i}\right)^{2}}
$$

where $n_{i}, n_{j}, k_{i}, k_{j}$, are the complex index of refraction of the media [4] , [5] .

Figure 2 shows a semi-transparent medium $\underline{2}$ limited by two dioptres on the interface between the media 1 and $\underline{2}$ and $\underline{2}$ and $\underline{3}$. Taking into account the effects of the internal transmittivity and of the multiple reflections on the interfaces [6], global coefficients of transmission and of reflection are found as follows:

$$
\begin{aligned}
& \mathbf{T}_{2}^{+}=\sum_{0}^{\infty}\left(1-\rho_{1,2}\right)\left(1-\rho_{2,3}\right) \tau_{2}^{2 n} \rho_{1,2}^{n} \rho_{2,3}^{n}=\frac{\left(1-\rho_{1,2}\right)\left(1-\rho_{2,3}\right) \tau_{2}}{1-\rho_{1,2} \rho_{2,3} \tau_{2}^{2}} \\
& \mathbf{R}_{2}^{-}=\sum_{0}^{\infty}\left(1-\rho_{1,2}\right)^{2} \tau_{2}^{2 n+2} \rho_{1,2}^{n} \rho_{2,3}^{n+1}=\frac{\rho_{1,2}+\tau_{2}^{2} \rho_{2,3}\left(1-2 \rho_{1,2}\right)}{1-\rho_{1,2} \rho_{2,3} \tau_{2}^{2}}
\end{aligned}
$$

It must be noted that in the opposite direction the coefficients are;

$$
\mathbf{T}_{2}^{-}=\mathbf{T}_{2}^{+} \quad \mathbf{R}_{2}^{+}=\frac{\rho_{2,3}+\tau_{2}^{2} \rho_{1,2}\left(1-2 \rho_{2,3}\right)}{1-\rho_{1,2} \rho_{2,3} \tau_{2}^{2}}
$$

Considering the radiant intensity emitted by an elementary surface in both positive and negative direction (figure 3) and combining the effects of the internal transmittivity and of the multiple reflections on the dioptres, the resultant radiant intensity on the boundary of the medium is in the positive direction;

$$
L^{+}\left(T_{2}\right)=\left\{\sum_{0}^{\infty}\left(1-\rho_{2,3}\right)\left(1-\tau_{2}\right)\left(1+\rho_{1,2} \tau_{2}\right) \rho_{1,2}^{n} \rho_{2,3}^{n} \tau_{2}^{2 n}\right\} L_{0}\left(T_{2}\right)
$$


http://dx.doi.org/10.21611/qirt.1994.006

where $\mathrm{L}_{0}\left(\mathrm{~T}_{2}\right)$ is the radiant intensity in the spectral band of the camera of the blackbody at the medium temperature. By integration in the half-space the radiative power emitted by the medium is;

$$
\mathrm{M}^{+}\left(\mathrm{T}_{2}\right)=\mathbf{E}_{2}^{+} \mathrm{M}_{0}\left(\mathrm{~T}_{2}\right)
$$

where $\mathrm{M}_{0}\left(\mathrm{~T}_{2}\right)$ is the radiative power emitted by the blackbody at the temperature $\mathrm{T}_{2}$, and the global emissivity coefficient of the semi-transparent medium is;

$$
\mathbf{E}_{2}^{+}=\frac{\left(1-\rho_{2,3}\right)\left(1-\tau_{2}\right)\left(1+\rho_{1,2} \tau_{2}\right)}{1-\rho_{1,2} \rho_{2,3} \tau_{2}^{2}}
$$

It must be noted that in the opposite direction the emission coefficient is;

$$
\mathbf{E}_{2}^{-}=\frac{\left(1-\rho_{1,2}\right)\left(1-\tau_{2}\right)\left(1+\rho_{2,3} \tau_{2}\right)}{1-\rho_{1,2} \rho_{2,3} \tau_{2}^{2}}
$$

Remark - The Kirchhoff law is verified;

$$
\mathbf{E}_{2}^{+}+\mathbf{R}_{2}^{+}+\mathbf{T}_{2}^{-}=1, \mathbf{E}_{2}^{-}+\mathbf{R}_{2}^{-}+\mathbf{T}_{2}^{+}=1
$$

and when $\rho_{1,2}=\rho_{2,3}$ the results obtained by Mac Mahon [4] are found.

\subsection{Radiative equations.}

Two main cases are useful for the present study.

3.3.1. An opaque body $\underline{3}$ with two semi-transparent media 1 and $\underline{2}$ (figure 4).

In this case the radiative power emitted in the direction of the camera is expressed by:

$$
\mathbf{M}=\mathbf{R}_{2}^{-} \mathbf{M}_{01}+\mathbf{E}_{2}^{-} \mathbf{M}_{02}+\left\{\frac{\varepsilon_{3} \tau_{2}\left(1-\rho_{1,2}\right)}{1-\rho_{1,2} \rho_{2,3} \tau_{2}^{2}}\right\} \mathbf{M}_{03}
$$

where $\mathrm{M}_{0 \mathrm{i}}$ is the radiative power emitted by the blackbody at the temperature of the medium $\mathbf{i}$ and the coefficient $\mathbf{R}_{\mathbf{2}}^{-}$and $\mathbf{E}_{\mathbf{2}}^{-}$are respectively given by the relations (3) and (8):

3.3.2. An opaque body $\underline{4}$ with three semi transparent media $\underline{1}, \underline{2}$ and $\underline{3}$ (figure 5 ).

In this case multiple reflections occur on three different boundaries of the media and

$$
\begin{array}{r}
\mathbf{M}=\left\{\mathbf{R}_{2}^{-}+\frac{\mathbf{T}_{2}^{-} \mathbf{T}_{2}^{+} \rho_{4} \tau_{3}^{2}}{1-\rho_{4} \mathbf{R}_{2}^{+} \tau_{3}^{2}}\right\} \mathbf{M}_{01}+\left\{\mathbf{E}_{2}^{-}+\frac{\mathbf{E}_{2}^{+} \mathbf{T}_{2}^{-} \rho_{4} \tau_{3}^{2}}{1-\rho_{4} \mathbf{R}_{2}^{+} \tau_{3}^{2}}\right\} \mathbf{M}_{02}+\left\{\frac{\mathbf{T}_{2}^{-}\left(1-\tau_{3}\right)\left(1+\rho_{4} \tau_{3}\right)}{1-\rho_{4} \mathbf{R}_{2}^{+} \tau_{3}^{2}}\right\} \mathbf{M}_{03} \\
+\left\{\frac{\varepsilon_{4} \mathbf{T}_{2}^{-} \tau_{3}}{1-\rho_{4} \mathbf{R}_{2}^{+} \tau_{3}^{2}}\right\} \mathbf{M}_{04}
\end{array}
$$

where $\mathbf{T}_{2}^{-}, \mathbf{T}_{2}^{+}, \mathbf{R}_{2}^{-}, \mathbf{R}_{2}^{+}, \mathbf{E}_{2}^{-}$and $\mathbf{E}_{2}^{+}$are given by the relations (2) to (4) and (7), (8):

\section{Calibration of the radiative coefficients of the materials}

The emissivity and the transmittivity of the materials have to be determined in the spectral band of the camera and under the incident angles encountered under the experimental conditions.

Equivalent radiative coefficients can be indirectly measured by means of:

- the infrared camera,

- auxiliary devices specially designed in order to reproduce the experimental conditions,

- the radiometric equations corresponding to the calibrations conditions.

These equivalent coefficients are only valuable in the tested particular conditions and for measurements carried out with the unchanged camera. 
http://dx.doi.org/10.21611/qirt.1994.006

\subsection{Atmosphere and environment}

The camera is placed at a distance of about $0.8 \mathrm{~m}$ from the object and the transmittivity of the atmosphere may be considered as equal to 1 .

The scanned area is isolated from eventual disruptive sources of radiation by black screens; at the ambient temperature. The radiative power emitted by the environment is suppos $ə d$ to be the one of the blackbody at the ambient temperature.

\subsection{Dicptres}

In the range of temperatures encountered $\left(20^{\circ} \mathrm{C}\right.$ to $\left.100^{\circ} \mathrm{C}\right)$ the index of refraction of the semi-transparent media remain constant and the reflectivities are:

- for the interface air/Calcium Fluoride $\rho=0.028$

- for the interface Calcium Fluoride/oil $\rho=0.0008$

\subsection{Calcium Fluoride}

The emissivity of this material does not depend on the direction [7]. In the spectral band of the camera, its internal transmittivity does not vary with the temperature between $20^{\circ} \mathrm{C}$ to $100^{\circ} \mathrm{C}[7],[8]$ and it is equal to 0.94 for a stator thickness of $12 \mathrm{~mm}[9]$.

\subsection{Graphite}

The auxiliary device used is composed of a sample of graphite of known temperature to which arget of emissivity equal to 0.99 is attached. The radiometric equation corresponds to the simpie case of an opaque body radiating in the atmosphere. It is found that the emitted radiant intensity is practically independent of the direction for an incident angle less than $60^{\circ}$ and that the emissivity remains close to 0.89 in the range of temperatures between $20^{\circ} \mathrm{C}$ to $100^{\circ} \mathrm{C}$.

\subsection{Oil}

In. f first device the oil is placed in a thermostatic container of hemispheric shape in such a manner that the depth of the oil is not dependent on the incident angle of measurement. It is verified that the emitted radiant intensity does not depend on the direction until an incidence angle of $60^{\circ}$.

In a second device the oil is placed in a graphite container formed by four concentric cavities whose depths are decreasing step by step from 0.13 to $0.025 \mathrm{~mm}$. The internal transmittivity of the oil is obtained from the equation $(10)$ where the media $1, \underline{2}, \underline{3}$ are respectively the atmosphere, the oil and the graphite. In addition;

- the temperature and the emissivity of the graphite container are known,

- the oil film is very thin and its temperature is supposed to be equal to the one of the bottom surface of the container.

For example the oil transmittivity $\tau_{2}$ is found to be comprised between 1 . and 0.91 in the temperature range $20^{\circ} \mathrm{C}-100^{\circ} \mathrm{C}$ for a thickness equal to $0.025 \mathrm{~mm}$.

\section{Validity of the method}

In order to test the proposed assumptions and to verify the measurements of the radiative coefficients, a device has been constructed which reproduces the experimental conditions (figure 6). This case corresponds to the equation (11) where:

- the Calcium Fluoride temperature is measured by means of a target of known emissivity attached to its surface, the temperature is supposed to be constant along the thickness of the plate,

- graphite and oil temperatures are equal and measured by using a thermocouple,

- the film thickness is fixed at a value of $0.1 \mathrm{~mm}$ by a calibrated support,

- the radiative coefficients are those previously measured. 
Infrared camera measurements of the graphite surface temperature were carried out in both transient and steady state regime. The relative difference of the thermocouple and camera results are less than $1.5 \%$ for values of the temperature from $75^{\circ} \mathrm{C}$ to $100^{\circ} \mathrm{C}$.

By attributing deliberately erroneous values to the parameters, the influence of these errors on the measurements can be shown. The parameters for which an inaccuracy is less consequent are:

- the temperature of the Calcium Fluoride because its transmittivity does not vary with the temperature,

- the temperature of the ambient medium because the radiative power emitted is small and it is attenuated by the factor $R_{2}^{-}$.

The parameters for which an inaccuracy has a large effect are:

- the values of the refraction index of the media,

- the transmittivity and reflectivity of the graphite; a variation of $5 \%$ of its emissivity leads to a relative variation of $1 \%$ of the measured temperature,

- the thickness and the transmittivity of the oil; the assumption of a transparent oil film leads to an over estimated temperature with a relative inaccuracy of about $2 \%$.

\section{Conclusions}

In a preliminary study [3] an approximate method was based on assumptions of a perfectly transparent oil film and Calcium Fluoride stator. In addition the determination of the graphite emissivity was not accurate enough. Influences of the parameters which have been pointed out in the present study permit the conclusion that the previous temperature measurements were over-estimated of about $5 \%$.

The method which has been analyzed in this paper was applied to mechanical face seals but the technique may be adapted to other types of hydrodynamically lubricated contacts such as those of bearings and thrust bearings.

\section{REFERENCES}

[1] FLOQUET (A.)- Températures de contact en frottement sec; détermination théorique et expérimentale. Thèse de Docteur Ingénieur, Université Claude Bernard, LYON, 1978.

[2] BORDENET - Approche de la thermique du contact élastohydrodynamique par une technique de mesure de rayonnement infrarouge. Thèse de troisième cycle, INSA Lyon, 1991.

[3] TOURNERIE (B.), REUNGOAT (D.), FRENE (J.) - Temperature measurements by infrared thermography in the interface of a radial face seal. Trans. $f$ the ASME, Joumal of Tribology, Vol.113, p571-576, July 1991.

[4] MAC MAHON (H.) - Thermal radiation from partially transparent reflective bodies. Journal of the Optical Society of America, Vol. 4, $n^{\circ} 6$, June 1950.

[5] BRUHAT (G.) - Cours de Physique générale - Optique. Masson Ed., 1965.

[6] SIEGEL (R.), HOWEL (JR.)- Thermal radiation heat transfer. Mc Graw Hill, 1981.

[7].TOULOUKIAN (YS.), DEWIT (DP.)-Thermophysical properties of matter. Vol.8, Purdue Research Foundation, 1972.

[8] HENRY (JF.) - Contribution à l'étude des transferts couplés conduction-rayonnement. Application aux protections thermiques. Thèse de troisième cycle, Université des Sciences Exactes et Naturelles de Reims, 1991.

[9] GAUSSORGUES (G.) - La thermographie infrarouge .Ed. Tecnique et Documentation, 1984. 
http://dx.doi.org/10.21611/qirt.1994.006

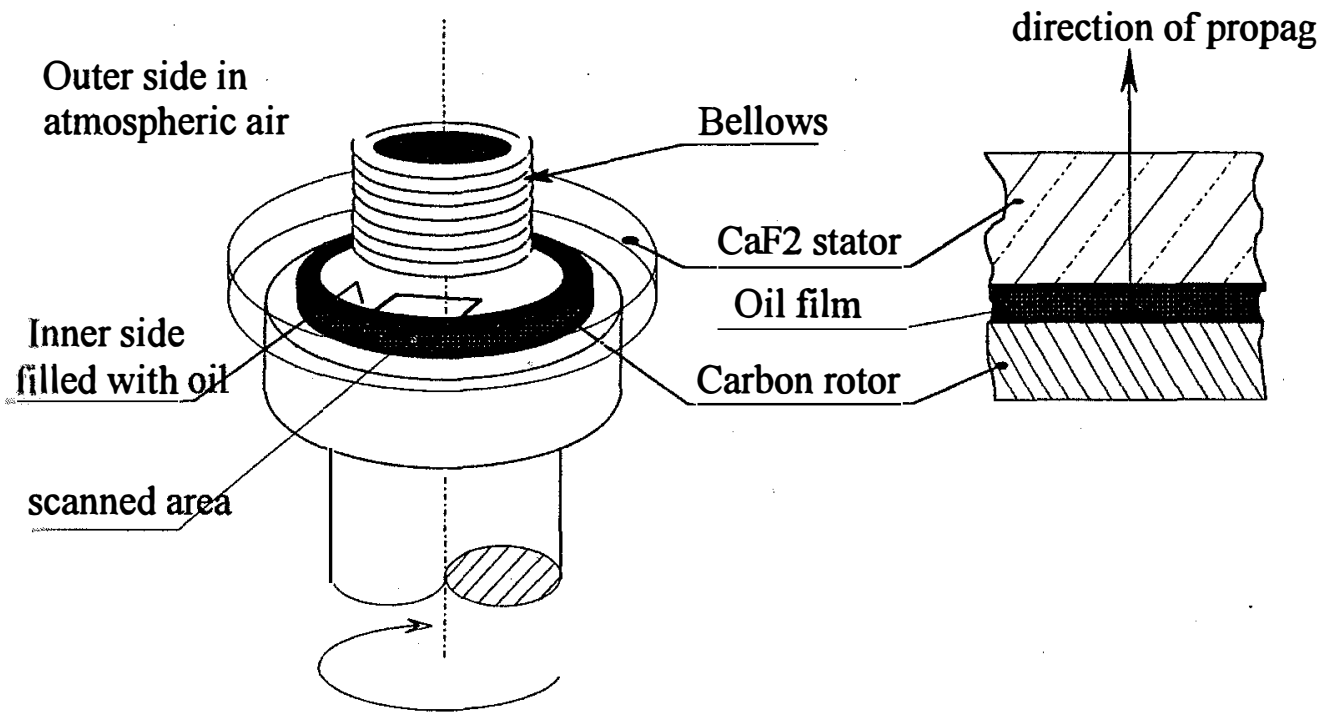

Fig.1. - Experimental seal and model

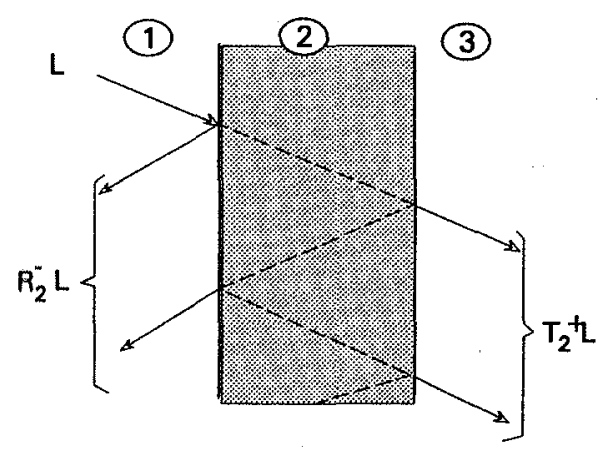

- direction of propagation

Fig .2. - Reflection and transmission in a semi-transparent medium

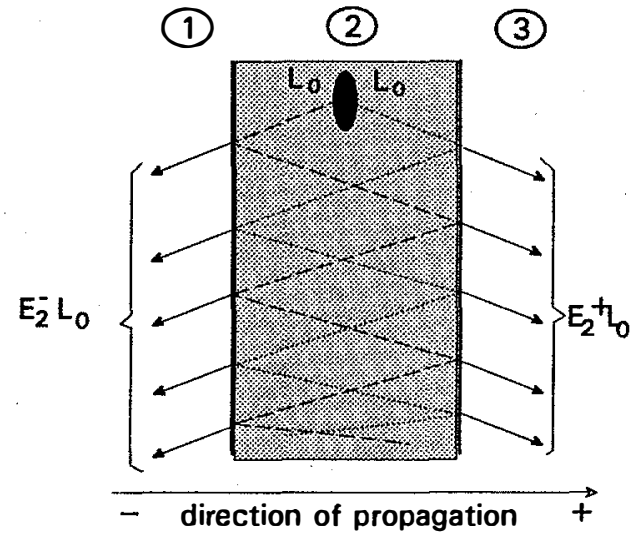

Fig .3. - Emission in a semi-transparent medium 
(1) (2) (3)

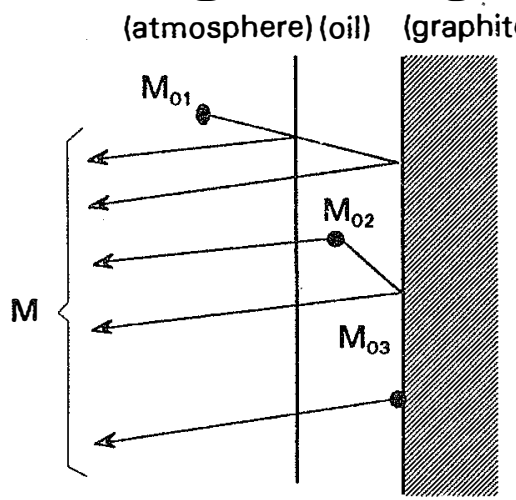

Fig. 4. - Opaque body and two semitransparent media.
(1) (2) (3) (4) atmosphere $\left(\mathrm{CaF}_{2}\right)$ (oil) (graphite)

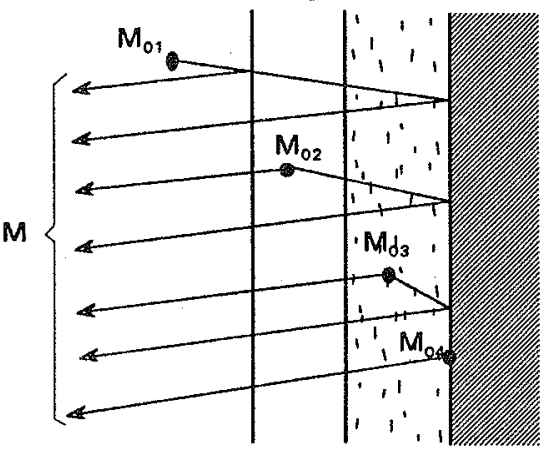

Fig. 4. - Opaque body and three semitransparent media.

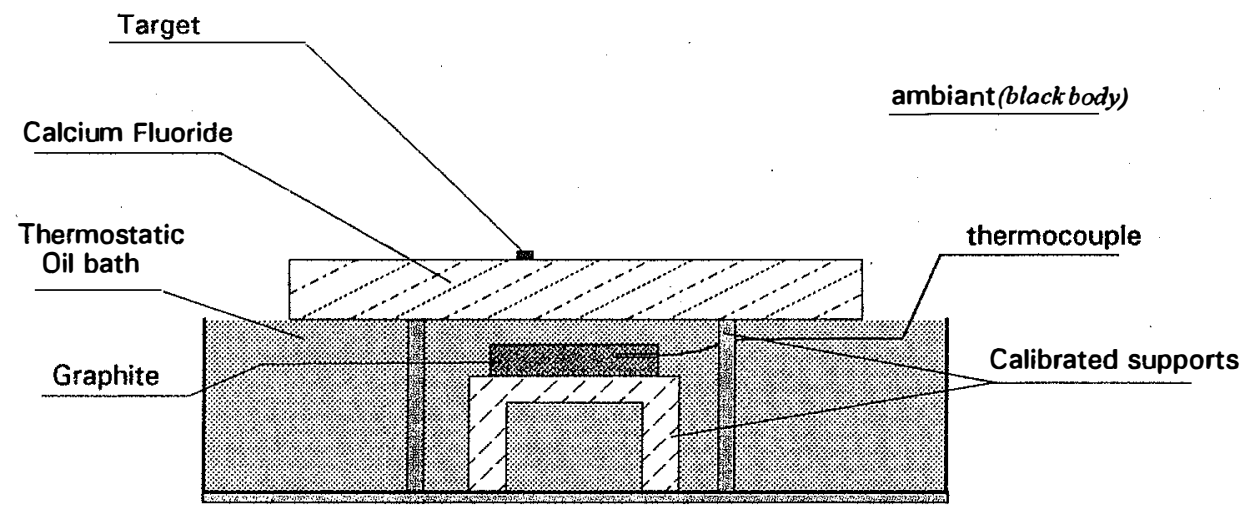

Fig. 6. - Set-up for verification. 